\title{
A Level Set Method Based on Bayesian Risk for Textured Image Segmentation
}

\author{
Yao-Tien Chen
}

\begin{abstract}
A level set method based on the Bayesian risk is proposed for textured image segmentation. First, the original texture image is converted into an orientation image. Second, based on Bayesian risk formed by false-positive and false-negative fraction in a hypothesis test, the level set evolution functional is deduced. Third, to prevent the propagating curves from generating excessively irregular shapes and lots of small regions, curvature and gradient of edges in the orientation image are integrated into the functional. Finally, to extract the boundaries of texture targets, the Bayesian level set equation is derived from the functional and then orientation image is regarded as input of the Bayesian level set equation. Compared with other segmentation methods for texture image, the proposed Bayesian level set approach relies on the optimum decision of pixel classification; thus the approach has more reliability in theory and practice. Experiments show that the proposed approach can accurately segment the texture image; moreover, the algorithm is extendable for multiphase segmentation.
\end{abstract}

Index Terms-Texture image, level set method, Bayesian risk, hypothesis test.

\section{INTRODUCTION}

The task of image segmentation/extraction is to partition the image into a number of regions such that each region has the same textural properties. Alternatively, this task can be seen as the problem of accurately extracting the borders (edges) between different texture regions in an image. This is one of the basic problems in image processing and pattern recognition. Techniques of textured image segmentation have been developed for many years. If a prior knowledge regarding the textural properties in a given image is available, then the problem is called supervised texture segmentation; otherwise it is called unsupervised. In this paper, we are concerned with the unsupervised texture segmentation method based on level set method.

The level set method was started by Osher and Sethian [1] in 1988. Since then, a great variety of geometric deformable models have been developed in response to the ever-increasing demands on image segmentation. Chan and Vese [2] proposed an active contour model working with no reliance on the gradient to stop the propagation process. With the stopping force based on Mumford-Shah segmentation formulas [3], the model becomes an energy-minimizing segmentation and given as

Manuscript received July March 25, 2017; revised August 15, 2017

The author is with the Department of Applied Mobile Technology, Yuanpei University of Medical Technology, HsinChu, 30015, Taiwan (e-mail: ytchen@mail.ypu.edu.tw).

$$
\begin{aligned}
\frac{\partial \varphi}{\partial t}= & \delta_{0}(\varphi) . \\
& {\left[\mu \operatorname{div}\left(\frac{\nabla \varphi}{|\nabla \varphi|}\right)-v-\lambda_{1}\left(g-c_{1}\right)^{2}+\lambda_{2}\left(g-c_{2}\right)^{2}\right], }
\end{aligned}
$$

where $g$ denotes the image gray levels, $\delta_{0}(\phi)$ is the Dirac measure (the derivative of the Heaviside function), $c_{1}$ is the average of $g$ inside the propagating curve, and $c_{2}$ is the average of $g$ outside the propagating curve; $\mu \geq 0, v \geq 0$, and $\lambda_{1}, \lambda_{2}>0$ are fixed parameters. Chan et al. also proposed active contours without edge for vector-valued images [4] and multiphase segmentation [5]. Multiphase level set frameworks can also be applied to motion segmentation. Based on the corresponding two-phase Chan-Vese model [5], Cremers [6] proposed a multiphase level set functional for segmenting multiple moving objects and moving background. The proposed functional depends on the velocity vectors for a set of disjoint regions and the boundary separating those regions. Segmenting the image plane into a set of regions of piecewise constant motion, this proposed variational approach can be considered an extension of the Mumford-Shah functional from the case of gray value segmentation to the case of motion segmentation.

Lee and Seo [7] proposed a level set-based partial differential equation $(P D E)$ based on the modified fitting term of the Chan-Vese model for the bimodal segmentation. The energy functional is designed to obtain a stationary global minimum; thus the energy functional has a unique convergence state, the evolution algorithm is invariant to the initialization, and level set function can set an appropriate termination criterion. Martin et al. [8] proposed a level-set active segmentation based on the maximum likelihood estimation to improve the segmented results for several different noise models and showed that the regularity term could be efficiently determined by using the minimum description length $(M D L)$ principle. They assume that noise can be described by members of the exponential family, such as Gaussian, Gamma, Poisson, or Bernoulli distribution.

Employing level set methods to perform texture segmentation is a hot study. Texture segmentation generally needs some extra preprocessing prior to the evolution processes, such as feature extraction and texture modeling. The goal of the feature extraction is to extract the most proper and representative features that will be used as inputs of the texture modeling; the texture modeling is used to find an appropriate model to represent texture image. Approaches to texture modeling are broadly classified into two classes; statistical modeling and modeling based on the filter theory.

Paragios and Deriche [9] proposed an improved Geodesic Active Contour model in which texture features are firstly 
extracted using Gabor filters, and then the propagating curves guided by both region-based and boundary-based forces move toward the final result. To clearly describe the method, the primary form of a two-phase segmentation is taken to explain. The active contour model is given as

$$
\begin{aligned}
\frac{\partial g}{\partial t}= & \alpha \log \left(\frac{P_{B}(I(g))}{P_{A}(I(g))}\right) N(g)+(1-\alpha)\left\{f\left[P_{C}(I(g))\right] k(g)\right. \\
& \left.-\nabla f\left[P_{C}(I(g))\right] \cdot N(g)\right\} N(g),
\end{aligned}
$$

where $g$ is a pixel of the curve $\partial R_{A}$ between regions $R_{A}$ and $R_{B}$; $I(g)$ denotes intensity of pixel $g ; P_{A}(I(g))$ and $P_{B}(I(g))$ are joint probabilities in $R_{A}$ and $R_{B} ; N(g)$ is the inward unit normal to $\partial R_{A} ; P_{C}(I(g))$ is a probability of boundary between $R_{A}$ and $R_{B}$; $f(\cdot)$ is a monotonically decreasing function; $\nabla f(\cdot)$ denotes gradient of $f(\cdot) ; k(g)$ is a curvature of $g$; and $0 \leq \alpha \leq 1$ is a constant. In this model, the first term is a region-based force shrinking and expanding the curve according to the strengths of joint probabilities $P_{A}$ and $P_{B}$, while the second term is a boundary-based force comprising two sub-terms; one shrinks the curve constrained by the curvature effect toward the object boundaries and the other attracts the curve to the object boundaries.

Aujol et al. [10] considered that textures are characterized by the energies of their wavelet coefficients in each wavelet subband. In the model, they simultaneously used spatial information and wavelet data to guide the curve evolution. A multiple functional consisted of partition, regularity, and data term is used for classification. Assuming that an image comprises $K$ texture regions and each region is completely determined by a level set function $\phi_{k}, k=1,2, \ldots, K$ (i.e., if a pixel $g$ belongs to region $k$, then $H\left(\phi_{k}(g)\right)=1$, where $H$ is the Heaviside function). The active contour model is given as

$$
\begin{aligned}
\frac{\partial \varphi_{k}}{\partial t}= & -\delta_{\alpha}\left(\varphi_{k}\right)\left[\lambda\left(\sum_{q=1}^{K} H_{\alpha}\left(\varphi_{q}\right)-1\right)\right. \\
& \left.-\gamma_{k} \operatorname{div}\left(\frac{\nabla \varphi_{k}}{\left|\nabla \varphi_{k}\right|}\right)+e_{k}\left(\sum_{i=1}^{I} B_{k}^{i}(g)\right)\right],
\end{aligned}
$$

with $B_{k}^{i}(g)=-\ln A_{k}^{i}+\ln 2+\frac{1}{2} \ln u_{i}(g)+\left(\frac{\sqrt{u_{i}(g)}}{\alpha_{k}^{i}}\right)^{\beta_{k}^{i}}$, where partition term, $\sum_{q=1}^{K} H_{\alpha}\left(\phi_{q}\right)-1$, penalizes the unclassified pixels and the pixels classified in two or more regions simultaneously; the data term, $\sum_{i=1}^{I} B_{k}^{i}(g)$, is the wavelet-based energy; the parameters $A_{k}^{i}, \alpha_{k}^{i}$, and $\beta_{k}^{i}$ are calculated from wavelets coefficients in texture modeling; $u_{i}(g)$ denotes the observed energies; $\delta_{\alpha}\left(\phi_{k}\right)$ is the Dirac measure; $\lambda, \gamma_{k}$, and $e_{k}$ are fixed parameters; and $i$ denotes the scale of wavelet packet decomposition.

In recent years, the bag-of-features ( $B o F \mathrm{~s})$ models have attracted increased attention in various domains ranging from texture retrieval to image segmentation. Li et al. [11] developed a patch-based method of pattern classification for dermoscopic images based on structure textons and a BoFs model. In their approach, the pattern structures of images are enhanced first; the enhanced lesion images with obvious directivity are then rotated to align principal directions with horizontal direction. The Otsu method is used to extract a region of interest and obtain patch vectors for each lesion image. For each pattern, the patch vectors of training lesion images are clustered to generate structure textons and a texton dictionary. Then, the $B o F$ s model is applied to obtain texton histograms for training and testing images, respectively. Finally, a nearest neighbor classifier with chi-square distance is adopted for classification.

The remaining sections of this paper are organized as follows. In Section II, we introduce the idea of image conversion which will be used to obtain orientation image and then develop our Bayesian level set method. In Section III, the experiments of texture segmentation are reported. Finally, conclusions are given in Section IV.

\section{THE PROPOSED METHOD}

The proposed segmentation method comprise two stages: image conversion and Bayesian level set method as shown in Fig. 1.

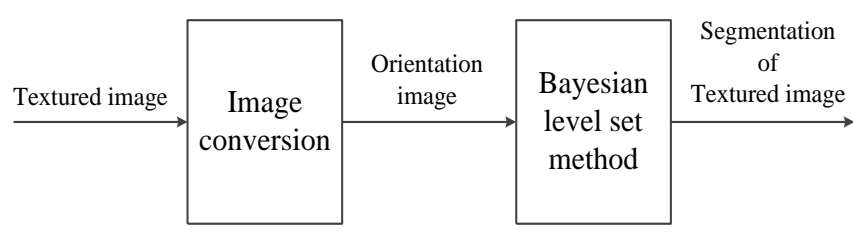

Fig. 1. The block diagram of the proposed method.

\section{A. Image Conversion}

Given a texture image, the preprocessing steps [12] for calculating the orientation image are given as follows:

Step 1: Divide the texture image into blocks of size $W \times W$.

Step 2: Compute the gradients $\partial_{x}(i, j)$ and $\partial_{y}(i, j)$ at each pixel.

Step 3: Estimate the local orientation of each block centered at pixel $(i, j)$ using the following equations:

$$
\begin{gathered}
V_{x}(i, j)=\sum_{u=1-\frac{W}{2}}^{i+\frac{W}{2}} \sum_{v=j-\frac{W}{2}}^{j+\frac{W}{2}} 2 \partial_{x}(u, v) \partial_{y}(u, v), \\
V_{y}(i, j)=\sum_{u=1-\frac{W}{2}}^{i+\frac{W}{2}} \sum_{v=j-\frac{W}{2}}^{j+\frac{W}{2}} \partial_{x}^{2}(u, v) \partial_{y}^{2}(u, v),
\end{gathered}
$$

and

$$
\theta(i, j)=\frac{1}{2} \tan ^{-1}\left(\frac{V_{y}(i, j)}{V_{x}(i, j)}\right)
$$

where $\theta(i, j)$ is the least square estimate of the orientation at the block centered at pixel $(i, j)$.

Step 4: Smooth the orientation field in local neighborhood using a Gaussian filter.

$$
\begin{aligned}
& \Phi_{\mathrm{x}}(i, j)=\cos (2 \theta(i, j)), \\
& \Phi_{\mathrm{y}}(i, j)=\sin (2 \theta(i, j)),
\end{aligned}
$$




$$
\begin{gathered}
\Phi_{x}^{\prime}(i, j)=\sum_{u=-\frac{w_{\Phi}}{2} 2}^{\frac{w_{\Phi}}{2}} \sum_{v=-\frac{w_{\Phi}}{2}}^{\frac{w_{\Phi}}{2}} W(u, v) \Phi_{x},(i-u w, j-v w), \\
\Phi_{y}^{\prime}(i, j)=\sum_{u=-\frac{w_{\Phi}}{2} 2}^{\frac{w_{\Phi}}{2}} \sum_{v=-\frac{w_{\Phi}}{2}}^{\frac{w_{\Phi}}{2}} W(u, v) \Phi_{y},(i-u w, j-v w),
\end{gathered}
$$

and

$$
O(i, j)=\frac{1}{2} \tan ^{-1}\left(\frac{\Phi_{y}^{\prime}(i, j)}{\Phi_{x}^{\prime}(i, j)}\right),
$$

here $\Phi_{x}$ and $\Phi_{y}$ are the $x$ and $y$ components of the vector field; $W$ is a two-dimensional low-pass filter with unit integral; $W_{\Phi} \times$ $W_{\Phi}$ specifies the size of the filter; and $O$ is the orientation after smoothing.

Step 5: Convert the orientation into gray level in each block, and then regard the gray-level image, called orientation image, as input of the Bayesian level set method.

\section{B. The Bayesian Level Set Method}

The Bayesian risk will be used to deduce the level set evolution functional for segmentation. An image is taken as a function $g(x, y): \Omega \rightarrow R$, where $(x, y)$ 's denote spatial coordinates and $\Omega$ is an open subset of $R^{2}$. The image is formed by two phases (zones) which may consist of several disconnected parts. We denote these phases as $\omega_{i}, i=1,2$, and the boundary of $\omega_{i}$ is $\partial \Omega$. Assuming that phase $\omega_{i}$ is represented by a Lipschitz function $\phi(x, y)$, called level set function, such that

$$
\begin{cases}\phi(x, y)<0, & \text { if }(x, y) \in \omega_{1}, \\ \phi(x, y)>0, & \text { if }(x, y) \in \omega_{2}, \\ \phi(x, y)=0, & \text { if }(x, y) \in \partial \Omega .\end{cases}
$$

We denote the evolving curve as $C$ and it is completely determined by level set function $\phi$. To describe a region in which $\phi$ is greater than zero or not, a unit step function called Heaviside function is used. The Heaviside function $H$ and its Dirac measure $\delta_{0}$ are defined as

$$
H(\phi)= \begin{cases}1, & \text { if } \phi \geq 0, \\ 0, & \text { if } \phi<0,\end{cases}
$$

and

$$
\delta_{0}(\phi)=\frac{d}{d \phi} H(\phi) .
$$

In the two-phase segmentation, the proposed approach is based on minimizing the functional containing Bayesian and regularity terms, and is described as

$$
F(C, \phi)=F_{B}(C, \phi)+F_{R}(C, \phi),
$$

where $F_{B}(C, \phi)$ is the Bayesian term and $F_{R}(C, \phi)$ is the regularity term; curve $C$ is represented by zero level set (i.e., $\phi(x, y)=0)$. The statistical decision theories are generally used for decision making. Here we apply minimizing the Bayesian risk to find the boundaries of targets in an image and the Bayesian term is defined as

$$
F_{B}(C, \phi)=\int_{\omega_{2}} P\left(\omega_{1}\right) P\left(g \mid \omega_{1}\right) d x d y+\int_{\omega_{1}} P\left(\omega_{2}\right) P\left(g \mid \omega_{2}\right) d x d y,
$$

where $P\left(\omega_{i}\right)$ is a prior probability of phase (zone) $\omega_{i}, i=1$, $2, \ldots, M, M$ is the number of phases to be segmented, $P\left(g \mid \omega_{i}\right)$ is the $p d f$ of pixel $g$ given phase $\omega_{i}$.

To prevent the curve from generating excessively irregular shape and lots of small regions, we set the regularity term as

$$
F_{R}(C, \phi)=v \int_{\Omega} \delta_{0}(\phi(x, y))|\nabla \phi(x, y)| d x d y,
$$

where $v \geq 0$ is the constant for weighting the regularity term.

Assuming that the gray levels of image pixels are Gaussian distribution and mutually independent (i.e., approximately independent and identically distributed). The $p d f$ of image pixels is expressed by

$$
P\left(g \mid \omega_{i}\right)=\frac{1}{\sqrt{2 \pi} \sigma_{i}} \exp \left\lfloor\frac{-\left(g-\mu_{i}\right)^{2}}{2 \sigma_{i}{ }^{2}}\right\rfloor, i=1,2,
$$

where $g$ denotes the random variable of pixel gray levels; $\mu_{i}$ and $\sigma_{i}$ are the mean and variance of phase $\omega_{i}$. To eliminate the exponential form of the Gaussian function, we take logarithm to make the functional of Bayesian term as

$$
\begin{aligned}
F_{B}(C, \phi) & =\int_{\omega_{2}} \ln P\left(\omega_{1}\right)-\frac{1}{2}\left(\ln \left(2 \pi \sigma_{1}^{2}\right)+\frac{\left(g-\mu_{1}\right)^{2}}{\sigma_{1}^{2}}\right) d x d y \\
& +\int_{\omega_{1}} \ln P\left(\omega_{2}\right)-\frac{1}{2}\left(\ln \left(2 \pi \sigma_{2}^{2}\right)+\frac{\left(g-\mu_{2}\right)^{2}}{\sigma_{2}^{2}}\right) d x d y .
\end{aligned}
$$

Based on finding the minimum extremal of the functional $F(C, \phi)$, the evolving curve $C$ will approach the target boundary. The functional $F(C, \phi)$ is minimized by solving the associated Euler-Lagrange equation. Consequently, the level set equation for evolution process is given as

$$
\begin{gathered}
\frac{\partial \phi}{\partial t}=\delta_{0}(\phi)\left\{v d i v\left(\frac{\nabla \phi}{|\nabla \phi|}\right)\right. \\
-\left\lfloor\ln P\left(\omega_{1}\right)-\frac{1}{2}\left(\ln \left(2 \pi \sigma_{1}^{2}\right)+\frac{\left(g-\mu_{1}\right)^{2}}{\sigma_{1}^{2}}\right)\right\rfloor \\
\left.+\left\lfloor\ln P\left(\omega_{2}\right)-\frac{1}{2}\left(\ln \left(2 \pi \sigma_{2}^{2}\right)+\frac{\left(g-\mu_{2}\right)^{2}}{\sigma_{2}^{2}}\right)\right\rfloor\right\},
\end{gathered}
$$

with initial condition $\phi(x, y, t=0)=\phi_{0}(x, y)$ in $\Omega$ and boundary condition

$$
\frac{\delta_{0}(\phi)}{|\nabla \phi|} \frac{\partial \phi}{\partial \vec{n}}=0 \text { on } \partial \Omega,
$$

where $\vec{n}$ is the unit normal at the boundary $\partial \Omega$ and $\frac{\partial \phi}{\partial \vec{n}}$ is the normal derivative of $\phi$ at the boundary.

In Eq. (20), there are two prior probabilities $P\left(\omega_{1}\right)$ and $P\left(\omega_{2}\right)$ which are still unknown. When a traditional method is used, it is generally assumed that the prior probabilities are equal for all phases. In contrast, we adopt a maximum likelihood estimation (MLE) [13] to estimate these unknown probabilities of each phase.

\section{EXPERIMENTS}

All algorithms were implemented in $\mathrm{C} \#$ programming 
language and MathLab library, and all experiments were performed on a general $P C$ with Intel Core i $7 \mathrm{GHz}$ processor and Microsoft Windows 7 operating system.

Three parts of the experiments are reported: calculating the orientation image, segmentation of texture image, and segmentation of triple-conjunction targets in texture image.

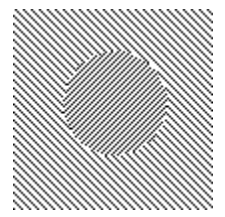

(a)

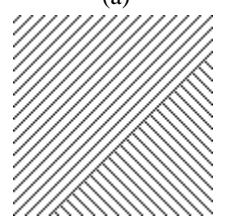

(d)

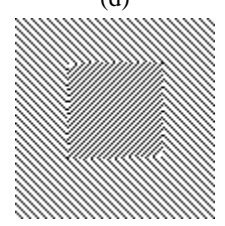

(g)

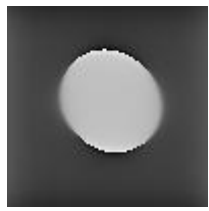

(b)

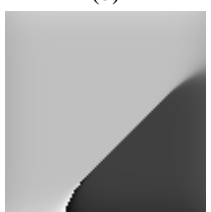

(e)

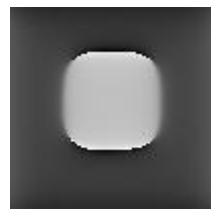

(h)

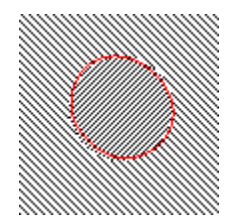

(c)

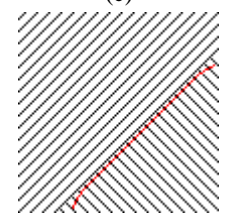

(f)

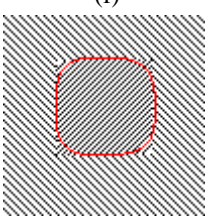

(i)
Fig. 2. Image conversion and segmentation in man-made texture images. (a), (d), and (g) The original man-made texture images. (b), (e), and (h) The orientation images. (c), (f), and (i) The results of the segmentation.

The proposed method was first used to segment the man-made texture images. As far as texture segmentation is concerned, we need a preprocessing to calculate the orientation image as detailed in the Section II.A before the level-set evolution processes. Three man-made texture images are shown in Fig. 2(a), (d), and (g). By means of the step of image conversion, we obtained the orientation images as shown in Fig. 2(b), (e), and (h). The segmented man-made texture images are shown in Fig. 2(c), (f), and (i).

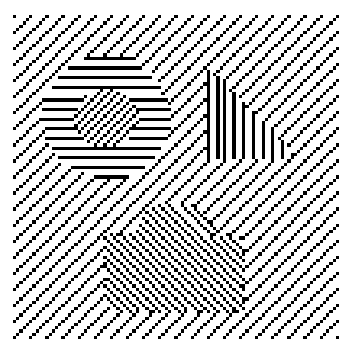

(a)

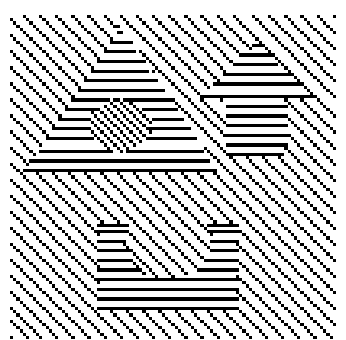

(c)

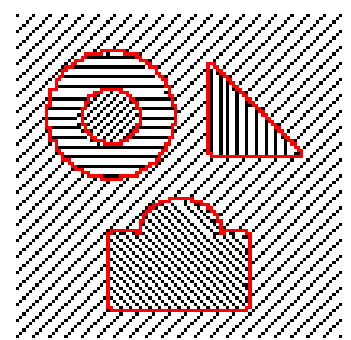

(b)

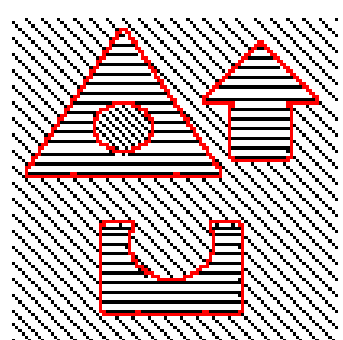

(d)
Fig. 3. Segmentation of man-made texture image. (a) and (c) The original man-made texture image with three different targets. (b) and (b) The results of the segmentation.

In the second experiment, the proposed approach was used to segment the man-made texture image with three different targets. As shown in Fig. 3, we can find that the proposed method can accurately achieve the segmentation.

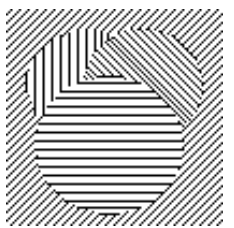

(a)

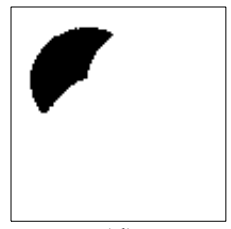

(d)

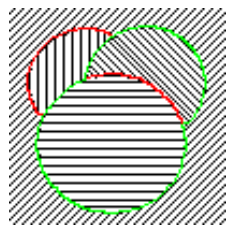

(b)

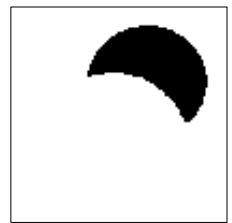

(e)

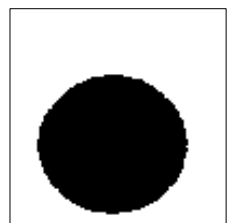

(c)

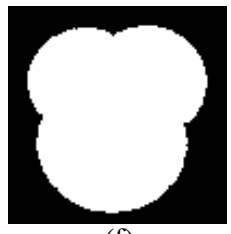

(f)
Fig. 4. Extraction of triple-conjunction targets in a man-made texture images. (a) The original man-made texture image. (b) The extracted result. (c) The extrcated region of $\phi_{1}>0$ and $\phi_{2}>0$. (d) $\phi_{1}>0$ and $\phi_{2}<0$. (e) $\phi_{1}<0$ and $\phi_{2}>$ 0. (f) $\phi_{1}<0$ and $\phi_{2}<0$.

In the next experiment, the proposed approach was extended for four-phase segmentation as one example shown in Fig. 4, where the proposed approach used two level set functions to extract triple-conjunction targets in a man-made texture image. Fig. 4(a) shows the original man-made texture image. Fig. 4(b) shows the extracted result. Figs. 4(d) to 4(f) show the extractions of different regions.

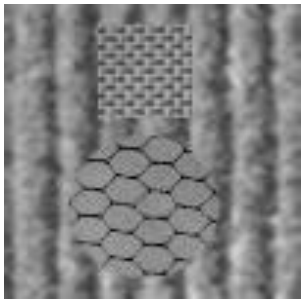

(a)

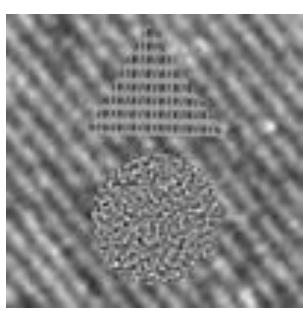

(c)

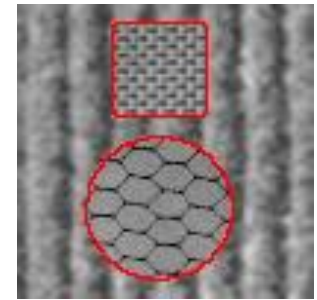

(b)

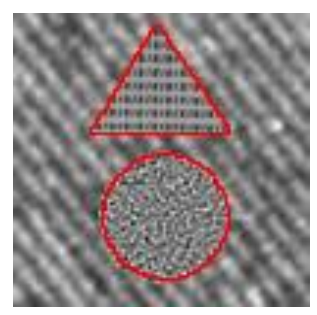

(d)
Fig. 5. Segmentation of the texture image. (a) and (c) The original texture image with two different targets. (b) and (b) The results of the segmentation.

In Fig. 5, the proposed approach was used to segment the real texture image. Figs. 5(a) and (c) show the original texture image with two different targets. Figs. 5(b) and (b) show the results of the segmentation. As indicated by the segmentation results, we can find that the proposed method can achieve the segmentation of texture image.

To verify the performance of the proposed method, we also examined the ability of texture extraction when the original texture image with triple-conjunction targets. Fig. 6(a) shows the original texture image with triple-conjunction targets. Fig. 6(b) shows the extracted result. Figs. 6(d) to 6(f) show the extractions of different texture regions. As revealed by the extracted results, the two-phase Bayesian level set proves to be capable of extracting boundaries of various texture objects. 


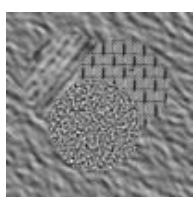

(a)

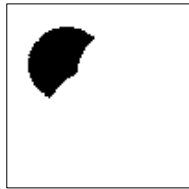

(d)

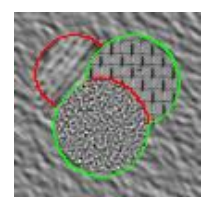

(b)

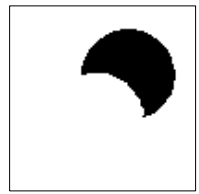

(e)

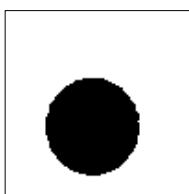

(c)

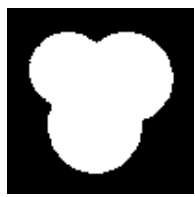

(f)
Fig. 6. Extraction of triple-conjunction targets in a texture image. (a) The original texture image. (b) The extracted result. (c) The extracted region of $\phi_{1}>0$ and $\phi_{2}>0$. (d) $\phi_{1}>0$ and $\phi_{2}<0$. (e) $\phi_{1}<0$ and $\phi_{2}>0$. (f) $\phi_{1}<0$ and $\phi_{2}$ $<0$.

\section{CONCLUSIONS}

In this paper, we proposed Bayesian level set method to segment the texture image. First, the texture image is converted into an orientation image. Second, based on Bayesian risk formed by false-positive and false-negative fraction in a hypothesis test and minimizing the average risk of decision in favor of the hypotheses, the level set evolution functional is deduced. Third, to prevent the propagating curves from generating excessively irregular shapes and lots of small regions, curvature and gradient of edges in the orientation image are integrated into the functional. Finally, to extract the boundaries of texture targets, the Euler-Lagrange formula is used to find the iterative level set equation from the functional.

We emphasize that the proposed method is equipped with the statistical analysis to estimate the boundary locations and region homogeneity; thus the proposed method possesses several extra advantages as compared with other level set methods: $(i)$. the conversion step exactly converts the texture image into an orientation image for benefit of Bayesian level-set evolution. ( $\mathrm{ii}$ ). the proposed approach takes the pixel distribution into the segmentation, so that the local noises have less influence. to the propagating process. (iii). the statistical decision theories are integrated into the derivation of level set method; hence the boundaries of texture targets can be more accurately extracted. (iv). the proposed method can be easily extended to facilitate the multiphase and 3-D surface segmentation

\section{REFERENCES}

[1] S. Osher and J. A. Sethian, "Fronts propagating with curvature-dependent speed: algorithms based on Hamilton-Jacobi formulations," Journal of Computational Physics, vol. 79, no. 1, pp. 12-49, 1988

[2] T. F. Chan and L. A. Vese, "Active contours without edges," IEEE Trans. Image Processing, vol. 10, no. 2, pp. 266-277, Feb. 2001.

[3] D. Mumford and J. Shah, "Optimal approximation by piecewise smooth functions and associated variational problems," Commun. Pure Appl. Math, vol. 42, pp. 577-685, 1989.

[4] T. F. Chan, B. Y. Sandberg, and L. A. Vese, "Active contours without edges for vector-valued images," Journal of Visual Communication and Image Representation, vol. 11, no. 2, pp. 130-141, June 2000.

[5] T. F. Chan and L. A. Vese, "A level set algorithm for minimizing the Mumford-Shah functional in image processing," in Proc. IEEE Workshop on Variational and Level Set Methods in Computer Vision, 2001, pp. 161-168.

[6] D. Cremers, "A multiphase level set framework for motion segmentation," in Proc. LNCS 2695, 2003, pp. 599-614.

[7] S. H. Lee and J. K. Seo, "Level set-based bimodal segmentation with stationary global minimum," IEEE Trans. Image Processing, vol. 15, no. 9, pp. 2843-2852, Sep. 2006.

[8] P. Martin, P. Refregier, F. Goudail, and F. Guerault, "Influence of the noise model on level set active contour segmentation," IEEE Trans. Pattern Anal. Mach. Intell., vol. 26, no. 6, pp. 799-803, June 2004.

[9] N. Paragios and R. Deriche, "Geodesic active regions and level set methods for supervised texture segmentation," International Journal of Computer Vision, vol. 46, no. 3, pp. 223-247, Mar. 2002.

[10] J. Aujol, G. Aubert, and L. Blanc-Feraud, "Wavelet-based level set evolution for classification of textured images," IEEE Trans. Image Processing, vol. 12, no. 12, pp. 1634-1641, Dec. 2003.

[11] Y. Li, F. Xie, Z. Jiang, and R. Meng, "Pattern classification for dermoscopic images based on structure textons and bag-of-features model," in Proc. LNCS 9219, 2015, pp. 34-45.

[12] L. Hong, Y. Wan, and A. Jain, "Fingerprint image enhancement: algorithm and performance evaluation," IEEE Trans. Pattern Anal. Machine Intell., vol. 20, no. 8, pp. 777-789, Aug. 1998.

[13] R. E. Walpole, R. H. Myers, S. L. Myers, and K. Ye, Probability \& statistics for engineers \& scientists, 9 th ed. New York: Prentice Hall, 2011, ch 9, pp. 307-312.

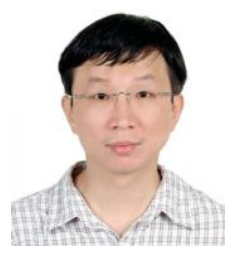

Yao-Tien Chen received his Ph.D. degree in computer science and information engineering from National Central University, Jhongli, Taiwan, in June 2007. He is currently an assistant professor in the department of applied mobile technology at Yuanpei University of Medical Technology, Hsinchu, Taiwan. His current research interests include somatosensory game design, image processing, and virtual reality; especially in the topics: medical image visualization, medical image segmentation, and wavelet-based image processing. 